Methods: 738 patients (age $62.0^{+} 11.1,84 \%$ women), followed a three weeks multidisciplinary program of individual and group sessions - with physiotherapy as main focus - during the period of August 2010 to September 2016 at Skogli Health- and Rehabilitation Center, Lillehammer, Norway.

3-month follow-up: $\mathrm{N}=252$ and 12-month follow-up: $\mathrm{N}=118$. Data from selfreported questionnaires at T1-T4 was gathered. Paired sampled T-tests and Pearson product-moment correlation coefficients was used to analyze the data obtained, using IBM SPSS Statistics v.23.

Instruments:

- NRS-11 for pain and stiffness at baseline (T1), at discharge (T2), and at 3- (T3) and 12 months (T4) after discharge.

- Likert scale (1-6) for self-rated level of health at T1, T2, T3 and T4.

- Self-reported level of training frequency at T1, T3 and T4

Results: There was a clear mean improvement $(p<0.0001)$ on all factors at T2 of moderate/large effect-size. At T3 there was a mean improvement $(p<0.05)$ on all factors, except pain, of small/moderate effect size. There was a mean improvement $(p<0.05)$ on self-rated level of health and training frequency at T4 of a small effect size. Worth noting is that the degree of stiffness and pain at T4 is back to T1-level.

There was a correlation $(p<0.05)$ between level of training frequency and selfrated level of health (small at T1/T3, medium at T4), but no correlation between level of training frequency and level of pain or stiffness, at any time. This suggests that a higher training frequency is associated with a higher sense of health regardless of symptom levels.

Conclusions: People with rheumatic disorders seem to have a very positive short term effect on all aspects after a three week intensive multidisciplinary program, but gradually return to pre-rehab levels during the following year - especially regarding symptoms like stiffness and pain. At the same time there seems to be a much slower decline in self-rated level of health - especially for those who regularly exercise. Properly randomized controlled trials are however needed to be able to draw any clear conclusions.

Implications: There might be a need for intensive multidisciplinary programs for rheumatic patients at intervals of less than a year, to be able to better keep the general health and function gained. Another possible implication is to implement a stronger focus on teaching rheumatic patients the necessity for an active lifestyle - including regular exercises - for them to be able to maintain their sense of general health, regardless of symptom levels.

References:

[1] Stoffer MA, Smolen JS, et al. Ann Rheum Dis 2015; 74: 1145-1149.

[2] Holm I, Tveter AT, et al. Physiotherapy 2015; 101 (3): 273-8.

Disclosure of Interest: None declared

DOI: 10.1136/annrheumdis-2017-eular.1258

\section{THU0732-HPR ENHANCED MANAGEMENT OF ANKYLOSING SPONDYLITIES THROUGH GUANGDONG INTERNET HOSPITAL IN CHINA:A RANDOMIZED, CONTROLLED TRIAL}

H. Zhengping ${ }^{1}$, L. Tianwang ${ }^{1}$, H. Zhixiang ${ }^{1}$, P. Xia ${ }^{1}$, Z. Lihua ${ }^{2}$, H. Yukai ${ }^{1}$ Y. Xiaoyan ${ }^{1}$, L. Meng ${ }^{1}$, H. Xuechan ${ }^{1}$, W. Yunqing ${ }^{1}$, L. Xumian ${ }^{1}$, T. Yao ${ }^{1}$.

${ }^{1}$ Department of Rheumatology and Clinical Immunology; ${ }^{2}$ Nursing Department, Guangdong NO.2 Provincial People's Hospital, Guangzhou, China

Background: Ankylosing Spondylitis (AS) is a kind of common chronic disease.Guangdong Internet Hospital is China's first officially recognized network hospital and the government encourage development of telemedicine in the country. Increasing research evidences support the efficacy of telemedicine in management of chronic diseases. However, There are still few researches about AS management by using telemedicine.

Objectives: We here conducted a 6-month randomized, controlled trial to evaluate the feasibility and efficacy of Guangdong Internet Hospital in AS management. Methods: A total of 102 AS patients were randomly divided into two groups: standard care (ST) group or standard care with Network-Enhanced Management (ST-NEM) group. NEM enhanced disease management including cognition of the disease, medication monitoring, behavioral management and psychotherapy. Individuals were assessed by using several tools at baseline and 6 months later: Bath Ankylosing Spondylitis Disease Activity Index (BASDAI) for the disease acticity, Ankylosing Spondylitis Functional Index (BASFI) for the functional limitation, the Zung Self-Rating Anxiety Scale (SAS) and the Zung Self-Rating Depression Scale Zung (SDS) for the psychological status, Pittsburgh sleep quality index (PSQI) for the sleep quality, and SF-36 for the general health status. In addition, we made a satisfaction survey about the network platform in the management of the disease. Both group received the same medications during the period. There were no significant differences in baseline demographic and clinical characteristics between the two groups.

Results: After 6 month, 91 patients completed the trial. BASFI $(1.75 \pm 0.73$ vs. $2.04 \pm 0.69, P=0.026)$, SAS $(28.12 \pm 3.22$ vs. $39.56 \pm 4.61, P=0.022)$, SDS (26.51 \pm 6.34 vs. $32.12 \pm 6.34, P=0.031)$, $P S Q I(3.31 \pm 0.46$ vs. $4.79 \pm 0.54, P=0.019)$ and SF-36 (SF-36M: $54.24 \pm 9.66$ vs. $61.41 \pm 8.56, P=0.014$; SF-36P: $63.42 \pm 11.08$ vs. $68.98 \pm 10.46, P=0.032$ ) were significantly lower in ST-NEM group than ST group after 6 months. There was no significant difference in BASDAI $(2.66 \pm 0.91$ vs. $2.75 \pm 0.75, P=0.068$ ) between the two groups. Individuals assigned to the ST-NEM group reported significantly improvement in functional limitation, psycho-
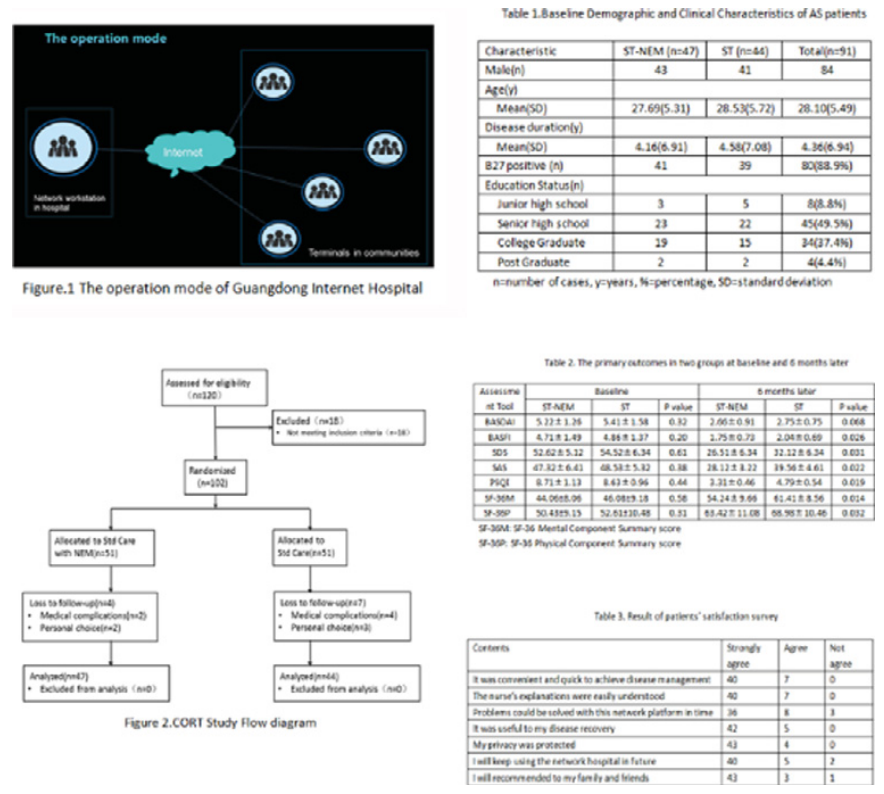

logical status, sleep quality and the general health status. In addition, Guangdong Internet Hospital were widely accepted by the AS patients in disease management. Conclusions: Guangdong Internet Hospital showed potential feasibility and efficacy in AS management. AS patients may receive disease management through the netwrok platform conveniently and effectively, especially those in the remote areas of the country. The results may be important for clinical practice in disease management of AS by using telemedicine.

References:

[1] Sinclair. 2015. Effectiveness and User Acceptance of Online Chronic Disease Management Interventions in Rural and Remote Settings: Systematic Review and Narrative Synthesis. Clinical Medicine Insights: Therapeutics: 43.

[2] Tu J, Wang C, Wu S. 2015. The internet hospital: an emerging innovation in China. The Lancet Global Health 3: e445-e6.

Disclosure of Interest: None declared

DOI: 10.1136/annrheumdis-2017-eular.2963

\section{THU0733-HPR REHABILITATION IN WARM CLIMATE FOR YOUNG ADULTS WITH INFLAMMATORY RHEUMATIC DISEASE. A 12 MONTHS RANDOMIZED CONTROLLED TRIAL}

I.R. Nilssen ${ }^{1,2}$, H. Koksvik ${ }^{2}$, K. Grønning ${ }^{1}$, A. Steinsbekk ${ }^{1}$. ${ }^{1}$ Department of Public Health and Nursing, Norwegian University of Science and Technology; ${ }^{2}$ Norwegian National Advisory Unit on Pregnancy and Rheumatic Diseases, Department of Rheumatology, ST.OLAVS Hospital, Trondheim, Norway

Background: Rehabilitation in warm climate has long been an established non-pharmacological treatment for patients with inflammatory rheumatic disease (IRD) in Norway. It has however not been tailored to the needs of young adults, who often have different challenges than older adults with IRD.

Objectives: The aim of this study was to investigate if a rehabilitation program in warm climate tailored to young adults from 20 to 35 years had effect on physical function and self-management/coping, 12 months after completed intervention.

Methods: This was an open randomized controlled pilot trial, with a 2-group parallel design and a 1:1 allocation ratio. Patients were recruited from three different rheumatology outpatient clinics in central Norway. The intervention group received a 17 day long rehabilitation stay in Spain. The main component of the tailored intervention was intensive exercise (2-3 times per/day), individual physiotherapy (daily) and patient education. The control group received treatment as usual. The primary outcome measure was physical function assessed by the "30 second Sit to Stand test" (30sSTS, number of sit and stand during 30 seconds, higher score is better) and self-management/coping measured by the "Effective Musculoskeletal Consumer Scale" (EC17, higher score is better).

Results: Forty patients (mean age 27.5, 65\% female) with IRD (intervention/control: $3 / 2$ rheumatoid arthritis, $3 / 9$ juvenile idiopathic arthritis, $4 / 5$ psoriatic arthritis, $8 / 3$ ankylosing spondylitis and $2 / 1$ polyarthritis) were randomized. 19 out of 20 patients completed the intervention. At twelve months follow up there were 3 patients lost to follow up from the intervention group, and 2 in the control group. Patients in the intervention group had a significant improvement in the 30sSTS test 3,6 and 12 months after completed intervention, compared to the control group (Table 1). The within group analysis showed that both groups improved at 6 and 12 months. The EC17 showed no difference between the two groups at 3 , 6 or 12 months.

Conclusions: The results indicate that the intervention group significantly improved their physical function one year after the intervention compared to the control group, but there was no effect on self-management/coping. These results 
Abstract THU0733-HPR - Table

\begin{tabular}{|c|c|c|c|c|c|c|c|}
\hline \multirow[t]{3}{*}{ Outcome measures } & \multirow{3}{*}{$\begin{array}{c}\text { Baseline values } \\
\text { Mean (SD) }\end{array}$} & \multicolumn{6}{|c|}{ Change from baseline to } \\
\hline & & \multicolumn{2}{|c|}{3 months } & \multicolumn{2}{|c|}{6 months } & \multicolumn{2}{|c|}{12 months } \\
\hline & & Mean $(95 \% \mathrm{Cl})$ & $p$-value & Mean $(95 \% \mathrm{Cl})$ & $\overline{p \text {-value }}$ & Mean $(95 \% \mathrm{Cl})$ & $p$-value \\
\hline \multicolumn{8}{|l|}{ 30sSTS } \\
\hline \multicolumn{8}{|l|}{ Within group } \\
\hline Intervention & $12.75(3.2)$ & $8.7(5.7-11.8)$ & $<0.001$ & $8.5(5.1-11.9)$ & $<0.001$ & $10.6(7.3-14.0)$ & $<0.001$ \\
\hline Control & $13.15(2.7)$ & $1.1(-0.5-2.7)$ & 0.169 & $3.7(1.2-6.2)$ & 0.006 & $3.8(0.6-7.1)$ & 0.024 \\
\hline Between groups & & $7.6(4.3-10.9)$ & $<0.001$ & $4.7(0.7-8.8)$ & 0.024 & $6.8(2.3-11.3)$ & 0.004 \\
\hline \multicolumn{8}{|l|}{ EC17 } \\
\hline \multicolumn{8}{|l|}{ Within group } \\
\hline Intervention & 64.5 (19.1) & $0.9(-4.0-5.7)$ & 0.709 & $2.2(-3.4-7.8)$ & 0.427 & $4.0(-3.7-11.7)$ & 0.291 \\
\hline Control & $64.4(10.3)$ & $2.2(-2.1-6.5)$ & 0.295 & $6.8(1.9-11.7)$ & 0.009 & $4.2(-1.2-9.5)$ & 0.119 \\
\hline Between groups & & $-1.3(-7.6-4.9)$ & 0.670 & $-4.6(-11.8-2.6)$ & 0.2 & $-0.2(-9.0-8.7)$ & 0.966 \\
\hline
\end{tabular}

might reflect that the focus of the intervention was mainly intensive exercise, and less on self-management /coping. This was a small study and the results should be interpreted with caution.

Disclosure of Interest: None declared

DOI: 10.1136/annrheumdis-2017-eular.2256

\section{THU0734-HPR ASSESSMENT OF LOCAL DISEASE ACTIVITY AFTER AN INTENSIVE HAND EXERCISE PROGRAM IN PATIENTS WITH RHEUMATOID ARTHRITIS MEASURED BY ULTRASOUND IMAGING: AN EXPLORATORY RANDOMIZED CONTROLLED TRAIL}

C. Bartholdy, M. Henriksen, E. Wæhrens, K. Ellegaard ${ }^{1,1}$. The Parker Institute, Frederiksberg, Denmark

Background: In $90 \%$ of patients with rheumatoid arthritis (RA) the joints of the hand are affected, causing impaired hand function. In general, reduced strength and range of motion of the hand are seen in patients with RA.

Studies have shown positive effect on pain and function after exercise intervention for the hand in patients with RA. However, it is unclear if the disease activity in the joints of the hand is influenzed by an exercise program.

Ultrasound imaging (US) is shown to correlate with other markers of disease activity in RA and can be used as a surrogate measure for inflammation in the joint, as US visualizes synovial hypertrophy and increased blood flow.

Objectives: To investigate if intensive hand exercise combined with joint protection education for the hand in women with RA can be conducted without a negative effect on the disease activity in the wrist and metacarpal (MCP) joints.

Methods: This is a sub-study of a randomised clinical trial investigating hand exercise therapy as add on to education in joint protection during activities of daily living (ADL) performance. The intervention group (IG) had both ADL education and exercise therapy and the control group (CG) had only ADL education.

The participants were women with RA involving the hand who had been on stable medication for at least three month.

At baseline all participants were examined by a rheumatologist. The joints were examined, blood samples collected and pain and strength of the hand were measured. Patient's functional ability was assessed using the Assessment of Motor and Process Skills (AMPS). US examination of the wrist and MCP 2-5 dorsal and volar in both hands was made. In the US examination both the amount of synovial hypertrophy and increased blood flow (Doppler activity) was evaluated. The evaluated was made according to a validated scoring system sum. (1)

After baseline examination the patients were randomised to either IG or CG. The hand exercise program contained both range of motion and strengthening exercises for the finger, wrist, elbow and shoulder joints. The exercise program was conducted four times a week for eight weeks, once a week supervised by a physiotherapist, to avoid overload of the joints and to progress the exercises if possible.

After eight weeks all baseline measures were repeated and change from baseline was calculated using the per protocol approach.

Results: Fifty five women with RA were included in the study (IG: 28; CG 27).

US score and CRP change from baseline:

\begin{tabular}{|c|c|c|c|c|}
\hline & $\begin{array}{l}\text { Intervention group } \\
(\mathrm{n}=22) \\
\text { Mean }(\mathrm{SD}) \\
\end{array}$ & $\begin{array}{l}\text { Control group } \\
(\mathrm{n}=25) \\
\text { Mean (SD) } \\
\end{array}$ & \begin{tabular}{|l|} 
Difference \\
Between groups \\
Mean $(95 \% \mathrm{CI})$ \\
\end{tabular} & $\begin{array}{l}P \\
\text { value }\end{array}$ \\
\hline $\begin{array}{l}\text { Baseline US } \\
\text { synovial } \\
\text { hypertrophy }\end{array}$ & $18.38(16.25)$ & $21.68(23.66)$ & & \\
\hline $\begin{array}{l}\text { Baseline US } \\
\text { Doppler }\end{array}$ & $6.57(12.48)$ & $7.24(13.15)$ & & \\
\hline $\begin{array}{l}\text { Baseline US } \\
\text { sum score }\end{array}$ & $23.82(27.18)$ & $28.92(35.97)$ & & \\
\hline Baseline CRP & $4.95(6.96)$ & $3.71(3.70)$ & & \\
\hline $\begin{array}{l}\text { Change US } \\
\text { synovial } \\
\text { hypertrophy }\end{array}$ & $0.57(3.93)$ & $1.8(7.16)$ & $-1.23(-4.74-2.28)$ & 0,36 \\
\hline $\begin{array}{l}\text { Change US } \\
\text { Doppler }\end{array}$ & $-0.33(4.62)$ & $0.92(8.20)$ & $-1.25(-4.28-1.77)$ & 0,52 \\
\hline $\begin{array}{l}\text { Change US sum } \\
\text { score }\end{array}$ & $0.23(6.90)$ & $2.72(13.63)$ & $-2.50(-7.74-2.76)$ & 0,43 \\
\hline Change CRP & $-0.86(6.13)$ & $1.86(3.71)$ & $-2.71(-5.67-0.23)$ & 0.10 \\
\hline
\end{tabular}

Eight withdraw during the study period, six from the $I G$ and two from the CG. Thus, 22 from the IG and 25 in the CG were included in the final analysis. The mean age 63.6 (12.6) years and mean disease duration was 12.7 (11.3) years, baseline mean tender and swollen joint were 4.8 (4.7) and $1.3(1.7)$, respectively. No differences in change from baseline in tender joint count, hand pain and strength and functional ability was seen between the two groups (data not shown). The mean score and change from baseline in US score and inflammatory marker $(\mathrm{CRP})$ in the blood are seen in table below.

Conclusions: Women with RA of the hand experience no negative effect on the disease activity in the joints of the hand after eight weeks combined hand exercise and joint protection education.

References:

[1] Hammer HB et al. Ann Rheum Dis 2011 Nov;70(11):1995-8.

Disclosure of Interest: None declared

DOI: 10.1136/annrheumdis-2017-eular.5193

\section{THU0735-HPR SHARED CARE OF RHEUMATOLOGIST AND NURSE CONSULTATIONS IN FOLLOW UP OF RHEUMATOID ARTHRITIS AND SPONDYLOARTHRITIS OUTPATIENTS WITH LOW DISEASE ACTIVITY: A MONOCENTRIC, RANDOMIZED CONTROLLED TRIAL}

K. Claes ${ }^{1}$, A. Van Hecke ${ }^{2}$, P. Jacques ${ }^{1} .{ }^{1}$ Rheumatology; ${ }^{2}$ Centre of nursery and midwifery, University Ghent, Ghent, Belgium

Background: Due to national regulations, the role of nurses in the management of arthritis patients differs significantly in all European countries (1). In Belgium, regularized consultations conducted by a rheumatology nurse do not exist.

Objectives: The study investigated whether rheumatology nurse consultations alternating with rheumatologist consultations for RA or AS patients with low disease activity is non-inferior compared to the usual care by the rheumatologist. Primary outcomes were safety and disease activity. Secondary outcomes were fatigue, pain, functional index, patient satisfaction, the level of self-management and self-efficacy.

Methods: This is a monocentric randomized controlled trial. The intervention group received a consultation conducted by a rheumatologist alternating with a nurse consultation, every 8 weeks. Patients in the control group received usual care: consultations performed by a rheumatologist every 8 weeks.

Results: Mixed method analyzes were performed. No statistical significant between-group effects were found nor in the RA group nor in the AS group, although there was a clinical relevance towards disease activity by AS patient in the intervention group. DAS 28 dropped with a mean difference of $0,4(-1,1-$ $-0,2$, SD 0,30). The ASDAS and BASDAI both decreased with $1.1(-1,4--1,3$, SD 0,1 and $-1,7--1,4$, SD 0,1 respectively). No safety signals from biochemical parameters such as SGOT, GOT and creatinine were detected. Inflammatory markers (ESR and CRP) remained stable in both groups. The secondary outcomes showed no significant between-group effects for both diseases. The self-efficacy outcome noticed a positive in-between-group effect by patients with $\mathrm{RA}$ in the intervention group.

Conclusions: In the follow-up of patients with RA or AS and low disease activity, outcome is not different when rheumatology nurse consultations alternating with rheumatologist consultations are compared to usual care. Implementing nurse consultations can positively influence the disease activity and patient self-efficacy. References:

[1] van Eijk-Hustings $Y$ et al. (2014) EULAR recommendations for the role of the nurse in the management of chronic inflammatory arthritis. Annals of Rheumatic Diseases 73:429-509.

Disclosure of Interest: None declared

DOI: 10.1136/annrheumdis-2017-eular.2341 